\title{
Trolley bus passengers' protection against accidental electrocution
}

\author{
I. Străinescu, V. Rădulescu, E. Tudor \& C. Unguraşu \\ ICPE SAERP S.A., Bucharest, Romania
}

\begin{abstract}
Assuring proper safety measures for all the passengers using the trolley, especially in the case of dangerous voltages, should be one of the most important issues to be taken into consideration. The method and the device that detects dangerous voltages that may appear between the body of the trolley and the road surface must be used on all types of trolleys that are supplied from a non-insulated network. The trolleys must be equipped with conducting bands or metallic cables - usually mounted close to the access doors - which collect the road potential. The device is supposed to monitor the potential difference between the body of the trolley and the road and detect two levels of dangerous voltage: the warning level, when the voltage is approximately $20 \mathrm{~V}$, and the disconnection level, when the voltage is over $40 \mathrm{~V}$, the case in which the main contactor is opened and the supply trolleys are lowered.
\end{abstract}

Keywords: trolley, danger, detection, potential.

\section{Introduction}

The risk of electrocution appears when the electrical insulation of the apparatus is broken and, during the entry of passengers, one of them is in touch with a conducting part of the trolley with his feet still on the ground. The dangerous voltage from which electrocution can appear is higher than $70 \mathrm{~V}$, but the protection level is stated at a maximum value of $40 \mathrm{~V}$. The possible voltage of the trolley body can be up to $750 \mathrm{Vdc}$, much higher than the upper limit.

There are different prevention methods and protection devices for passenger protection against electrocution during the use of the trolleybus. The prevention measures ensure better insulation of the electrical apparatus, special construction of those devices with double-step insulation, periodically testing of the insulation 
and special materials used to build the doors of the trolleybuses. The protection devices must continuously check the body-to-ground voltage and to disconnect instantaneously the trolleybus from the power line [1].

In order to assure efficient protection, the fact that Bucharest has a trolley park of 300 trolleys, most of them having chopper drive and a d.c. motor, and some of them having three-phase inverter and asynchronous traction motor, was taken into consideration. For such a large park, assuring efficient protection in case of dangerous voltage on the trolley's body is very important.

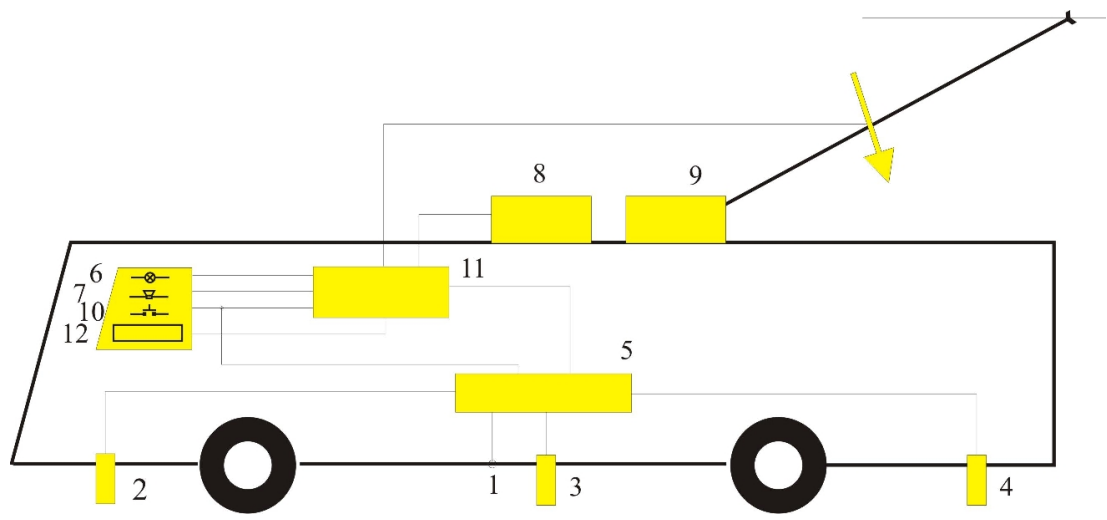

Figure 1: $\quad$ Electrical principle diagram - trolley.

\section{Example of a protection device}

The signalisation and protection device consists of sensor STP 5 and the command unit of the trolley 11 . The control unit 11 contains master and slave microcontrollers, memories, and provides diagnosis facilities.

The voltage measurement between the body of the trolley and the road surface uses three conducting bands (usually made of rubber carbon impregnated bands with copper core) - mounted close to the access doors. Basically, the STP device has a voltage transducer to measure the voltage that can accidentally appear at the body of the vehicle.

When the STP5 detects a potential difference bigger than the warning voltage (threshold 1 is usually set at 20V), it sends a signal to the trolley's desk by turning on a warning lamp 6 and by activating a buzzer 7 . If the phenomenon persists, the driver can decide to return the trolley to the garage.

If the phenomenon gets worse and the measured voltage surpasses a given value defined as the protection threshold, (value is chosen between 40 and $70 \mathrm{~V}$ ), the controller will disconnect the main switch 8 of the trolley and also will retract the trolley poles 9 away from the contact lines. The main switch turn-off time is usually $5 \ldots 100 \mathrm{~ms}$, and if the insulation failure on the body isn't located on the link between the current collectors and the main switch, the dangerous voltage will immediately disappear. Based on the trolley pole retreat, the disconnection time is around 2 seconds. 
From the passenger protection safety analysis, the power cable installed between the current collector 9 and the main switch 8 must be checked for deterioration at a certain period of time.

During an insulation failure, lamp 6 on the trolley's board will flicker and the buzzer 7 will vibrate with a small frequency of $1 . .5 \mathrm{~Hz}$, warning the driver of the situation. The cabin warnings are presented on a digital display 12 , indicating the cause of the malfunction, the location and the cause of disconnection.

\section{Protection method}

The protection method involves a test of the whole protection system which includes measuring device 5 , conducting bands 2,3 or 4 , the electric connection to the trolley's body and the links between them, by pressing a testing button 10 .

The method also involves a continuous validation of the STP5 device, so that when malfunction occurs on the road/in exploitation, the main switch and the current collector from the contact line shall automatically disconnect.

If the warning still persists after the disconnection, the STP5 must be declared broken and usually the trolley will be taken to the garage.

After the line 9 current collectors have been disconnected and the malfunction warning has stopped, the driver will have to ask for intervention and be towed to the garage. Usually, while at the station, reconnecting to the network is not allowed.

The protection method implies that the whole protection system should be verified, including the device 5 , conducting bands or metallic bands $2,3,4$, the electrical link to the trolley's body and the links between them, by pressing a button 10. The method also implies a continuous auto testing of the STP 5 device, so that when it malfunctions during operation, the main switch and contact line collector automatically disconnect. If after the disconnection, the malfunction warning doesn't stop, that means that the STP 5 device malfunctioned, and the trolley will be towed back to the garage.

\section{Risk analysis}

Final Event A, according to [2], depends on the occurrence of the following events at the same time (AND function):

- Event B (Code 1100) The body-to-ground voltage is bigger than 40 $\mathbf{V}$ (insulation is broken);

- Event C (Code 1200) The trolley poles 9 are connected to the overhead line, and the main switch 8 is on (trolley bus is $\mathrm{ON}$ );

- Event D (Code 1300) The driver or one of the passengers has one foot touching the ground and an arm touching the body of the trolley (if the trolley is in station and the door is open).

Events B, C and D also depend on a series of lower level events according to the OR logic: 


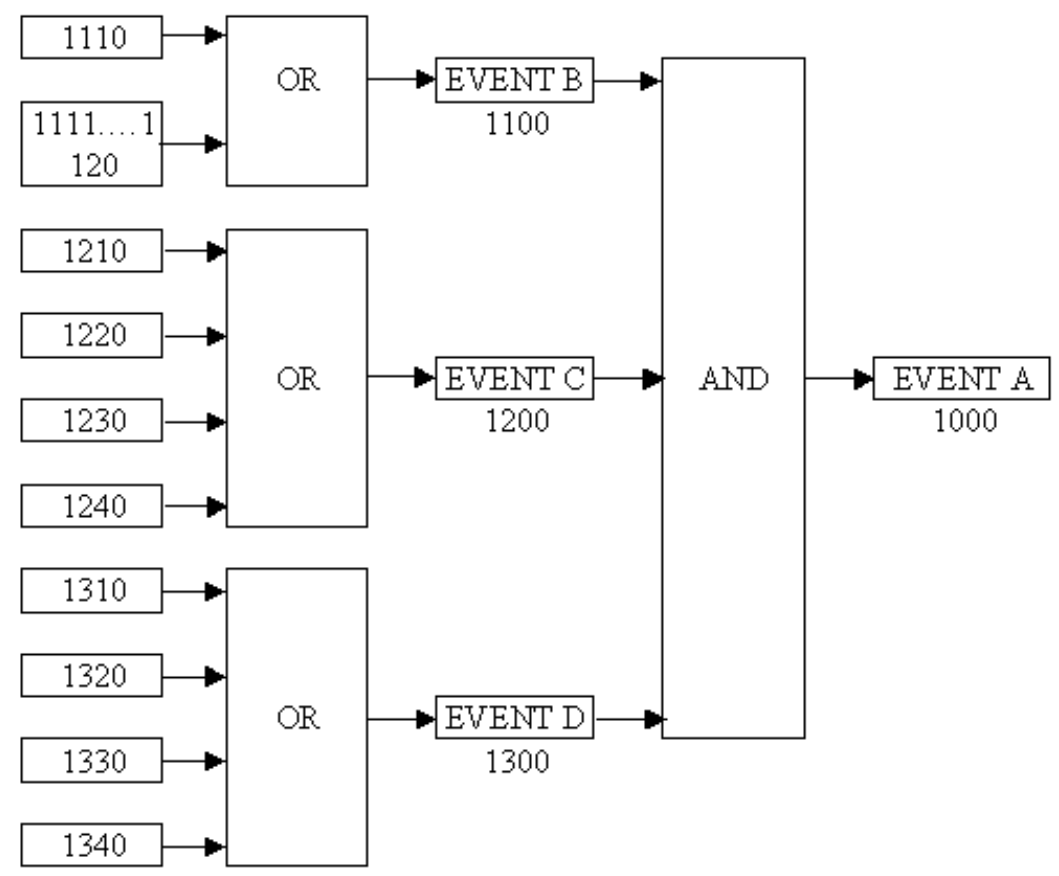

Figure 2: Risk analysis diagram.

- Event B depends on these events: code 1100 - The insulation is deteriorated (two steps insulators from the same equipment deteriorated (Fig 2. - example - event 1111= line filter insulation);

- Event $\mathrm{C}$ depends according to the OR logic on a series events that also depend on the passenger and driver safety method:

- The STP5 device doesn't function = event code 1210;

- The driver disconnects the STP5 device (code 1220);

- The collectors $(2,3,4$ on figure 1$)$ are deteriorated (Code 1230);

- Ground connection missing (no 1 on figure 1) (Code 1240).

- Event D depends according to the OR logic on these events:

- When the driver is being warned of the occurrence of dangerous voltage and if the main switch doesn't disconnect and at the same time if the trolley poles do not retreat (code 1310).

○ The door insulation failure (code 1320).

- A passenger touches the body of the vehicle (code 1330).

The next step to determine the proposed method of safety is identifying the occurrence risk of event $\mathrm{A}$ when the actual signalling and protection device exists. 


\section{Convention:}

$\boldsymbol{\Lambda}$ - event occurrence intensity per operating hour;

TMIDE - medium time between two events; equals $1 / \Lambda$; this parameter pretty accurately suggests the probability of an event.

AND function: The event A's TMIDE is equal to the sum of the medium time of events B, C, and D, fact that we can observe from figure 3 , due to the AND function from entering event $A$.

$$
\operatorname{TMIDE}(\mathrm{A})=\mathrm{TMIDE}(\mathrm{B})+\mathrm{TMIDE}(\mathrm{C})+\mathrm{TMIDE}(\mathrm{D})
$$

OR function: The probability $\Lambda(\mathrm{X})$ of events $\mathrm{B}(\mathrm{Xi}), \mathrm{C}(\mathrm{Xi})$ and $\mathrm{D}(\mathrm{Xi})$ can be determined with the general relation:

$$
\Lambda(\mathrm{X})=\Lambda(\mathrm{X} 1)+\Lambda(\mathrm{X} 2)+\Lambda(\mathrm{X} 3)
$$

\section{Event A, Event code 1000: Driver or passenger electrocution}

This event is one of the most dangerous that can appear operating a trolley bus. It refers to the danger described in 2.2, annex A - SR EN 1050 / 2000: Electrical dangers due to human contact with parts that have become active after malfunction (indirect contact).

Event A's medium time is equal to the sum of the medium time of events B, C and $\mathrm{D}$, due to AND function from entering event $\mathrm{A}$.

Event B, Event code 1100: The body's voltage compared to the ground is bigger than $40 \mathrm{~V}$ (deteriorated insulation between the body and the $750 \mathrm{Vcc}$ line voltage)

This event takes place if the insulation from step 1 and 2 of high voltage is deteriorated or if the STP device doesn't function normally or if despite the command, the main switch hasn't disconnected. We have to observe that even if the main switch disconnects, if the insulation is deteriorated (linking the collectors to the main switch), the dangerous voltage persists because there aren't any automatic trolley retrievers that should disconnect on the command of the STP device.

$$
\begin{aligned}
& \Lambda(\mathrm{B})=\Lambda(\mathrm{E})+\Lambda(\mathrm{F})+\Lambda(\mathrm{K}) \\
& =0,105 \times 10^{-6}+0,015 \times 10^{-6}+0,12 \times 10^{-6}=0,24 \times 10^{-6} \\
& \text { TMIDE }(\mathrm{B})=1 / \Lambda(\mathrm{B})=1 / 0,24 \times 10^{-6}=4,1 \times 10^{6} \text { hours }
\end{aligned}
$$

Event C, Event code 1200: Polarity trolley $(+)$ is on the line and the main switch is connected.

This event occurs when the STP device detects a dangerous voltage of over 40 $\mathrm{V}$, but the high voltage contactor doesn't disconnect, or the malfunctioning STP doesn't notice the dangerous voltage, or the trolley pole doesn't lower.

$$
\begin{aligned}
& \Lambda(\mathrm{C})=\Lambda(\mathrm{F})+\Lambda(\mathrm{G})+\Lambda(\mathrm{U}) \\
& =0,015 \times 10^{-6}+3,315 \times 10^{-6}+5 \times 10^{-6}=8,33 \times 10^{-6}
\end{aligned}
$$

$\operatorname{TMIDE}(\mathrm{C})=1 / \Lambda(\mathrm{C})=1 / 8,33 \times 10^{-6}=1,05 \times 10^{6}$ hours 
Event D, Event code 1300: The driver or one of the passengers has one of their limbs touching the ground and another touching the outside body of the trolley or an access step

The event appears when the trolley is at the station and one of the passengers is touching the trolley's body with a part of his body (most probably the hand) and has one leg on the ground.

This event could also appear when a passenger has one leg on the ground and one hand on the door's handle, which has deteriorated insulation or the other leg it's on an access step with a deteriorated insulation.

Another possibility may involve the driver, who, even if the automatic switch has disconnected, the insulation defect in before the switch and if the driver on his way to pull down the collectors doesn't jump right out of the trolley or doesn't pay attention and touches the trolley's body.

$$
\begin{gathered}
\Lambda(\mathrm{D})=\Lambda(\mathrm{S})+\Lambda(\mathrm{T})=0,15 \times 10^{-6}+0,15 \times 10^{-6}=0,3 \times 10^{-6} \\
\operatorname{TMIDE}(\mathrm{D})=1 / \Lambda(\mathrm{D})=1 / 3 \times 10^{-6}=3,3 \times 10^{6} \text { hours }
\end{gathered}
$$

For the Event A determination we use the values resulted from equations (4), (6) and (8) in (1):

$$
\begin{aligned}
& \text { TMIDE(A) }=\mathrm{TMIDE}(\mathrm{B})+\mathrm{TMIDE}(\mathrm{C})+\mathrm{TMIDE}(\mathrm{D}) \\
& =4,1 \times 10^{6}+30,05 \times 10^{6}+3,3 \times 10^{6} \text { hours }=37,45 \times 10^{6} \text { hours. }
\end{aligned}
$$

Considering that, for a big city like Bucharest, with a park of 300 trolleys, and that one trolley can run maximum $20 * 300$ days $=6.000$ hours / year, for 20 years we have:

$$
\operatorname{TMIDE}(\mathrm{A})=6.000 * 300 * 20=36 * 10^{6} \text { hours, }
$$

which represents (shows) that there is the probability of less than one event $\mathrm{A}$ occurring in these 20 years. From the calculations the TMIDE (C) should be $30 * 10^{6}$ hours, which is a big value.

Without the STP 5 device, this event would occur with a probability of once every 4 years, because the TMIDE (C) equals $1,2 \times 10^{6}$ hours, a fact that isn't acceptable for a park as large as the one in Bucharest.

\section{Improving the detection unit by using the risk analysis}

A better protection for passengers is assured when the medium time between two consecutive errors is higher. To increase this time, the following measures must be applied:

- The driver cannot have the possibility to deactivate the STP5, under any circumstances. For previous versions, the driver could deactivate the STP because of the false signalling as lightning or crossroads between tramway and trolley lines. 
- The STP 5 must be a self-checked device, in order to avoid internal failure.

- When the STP 5 is defective, the main switch 8 will be switched off and the trolley poles 9 will retreat and will signal a STP 5 malfunction. The trolley will be towed to the garage and the malfunctioned STP 5 will be replaced.

- $\quad$ The device STP 5 will have higher reliability, by using high quality materials and by increasing the measuring procedures.

- According to the proposed safety method, a set of supplementary measures will be taken, to verify the insulation status:

○ Daily, the trolley stops at the exit gate of the garage with the three collectors (no. 2, 3 and 4 on figure 1) on a metallic plate, where the test procedure must be fulfilled: The test button 10 will be pushed and, consequently, a voltage of $30 \mathrm{Vdc}$ will be applied on the measuring chain, and the continuity of the circuits will be checked, by measuring the current that runs through the measuring circuit and through the collectors, as well. The test voltage is smaller than the dangerous voltage $(40 \mathrm{Vdc})$, but higher than the signalization threshold (20Vdc). During this test the lamp and the buzzer can be checked, too. The test shall be recorded in the non-volatile memory.

- It is recommended the continuity of the conducting bands to be checked at the ends of the line, by placing a metallic plate of a corresponding length.

- According to the safety method, periodical revisions will be done, for the two insulation steps on the entire electrical equipment and especially for the electrical circuit between the pantograph and the main switch; the reason behind is that the time it takes for the trolley to retreat from the line is 10 times higher compared to the time it takes for the main switch to open the circuit; therefore the is a small probability that the dangerous voltage should appear due to a malfunction on this circuit and until it disconnects from the line, a passenger from outside the trolley could find himself under voltage if the malfunction occurs while the trolley is in the station.

As stated in the safety method, in order to assure a proper operation, the continuity of the collector bands 2,3 and 4 must be checked when leaving the garage and at the ends of the line (Fig $3 \mathrm{~B}$ and $3 \mathrm{C}$ ). For better result of the test, place the trolley upon a metal plate so that, at the same time bands 2, 3 and 4 will be in contact with the plate. Pushing test button 10 (Fig.2) will transmit a test command to the logic block 11, which will also send sequential commands to the switch 15 which configures the diagram as described in figure $3 \mathrm{~B}$ and $3 \mathrm{C}$, as the collecting bands will be connected in series two by two. The voltage is provided from the DC- DC converter 14, conducted through the collectors 2, 3 and 4, through the metal plate and applied to the rectifier 16 and transducer 17. 


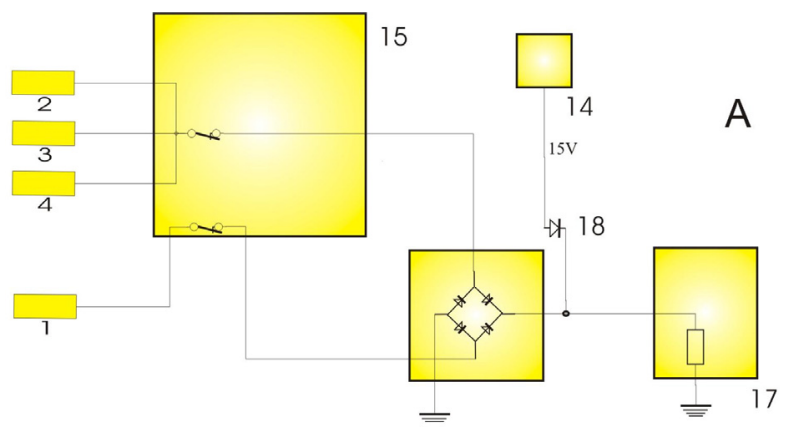

16

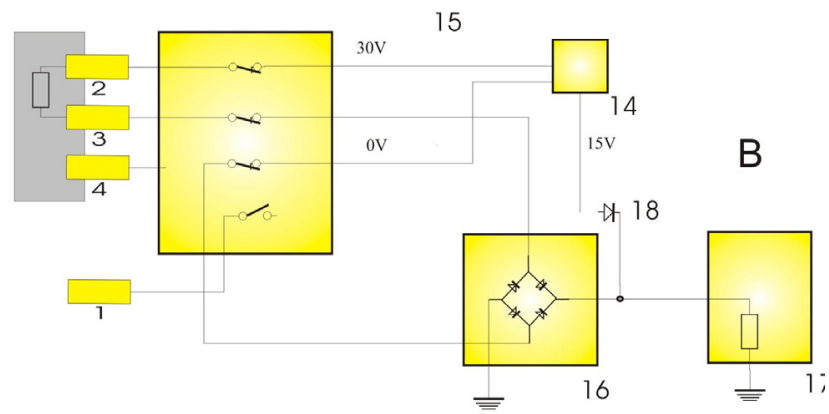

15

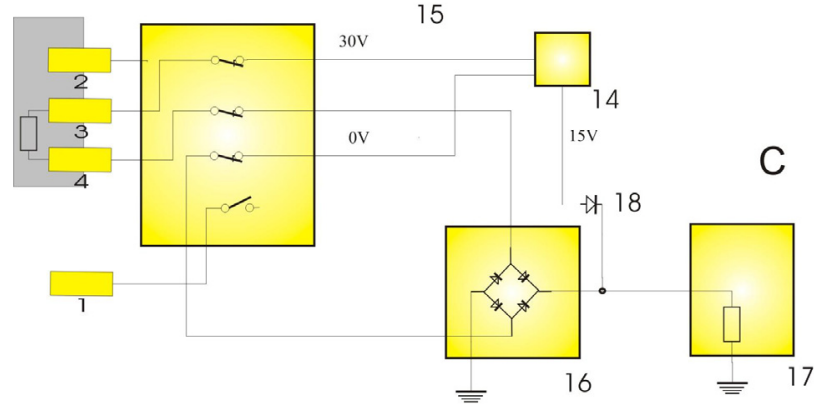

Figure 3: Different configuration of the STP diagram.

In this manner, if the current that runs through the voltage transducer has a proper value, determined by a $30 \mathrm{Vcc}$ test voltage and the resistor from the transducer's primary, for both diagrams results in the integrity of the collector bands, including their electrical links to the main parallel-series controller 14.

\section{Conclusions}

The protection against electrocution is highly vital, as the occurrence of such accidents was very important in the past, including also deadly casualties. In 
order to avoid such accidents, a deep analysis, shortly described within this paper, regarding the electrocution risk by indirect touch was conducted. The conclusions of this analysis are:

1. The double insulation of the vehicles must be checked periodically.

2. The maintenance of the trolley poles and of the cables connected within them and the main circuit breaker must contain the obligation of change them regularly.

3. The collectors $(2,3$ and 4$)$ that are used to capture the ground potential must be checked daily.

4. A new device must be produced to capture the dangerous potential of the bus body reported to the ground, with two thresholds: first, the signalization and the second the disconnection of high voltage. This device has permanent self-testing and cannot be switched off.

5. When a double insulation is broken and the dangerous voltage is connected to the body, the device must act quickly in order to disconnect the high voltage by both disconnections of the main circuit breaker and retreat of the trolley poles.

By using these conclusions in designing the STP protection device, the calculation of the average data between electrocution accidents is estimated at 20 years, for a park of 300 vehicles. This analysis is implemented right now on the RATB vehicles equipped with the power electronics produced by ICPE SAERP S.A.

\section{References}

[1] E/ECE/TRANS/505 Add no.36, published on E/ECE/324 Revision/ Addendum35/Revision2 Amedt1.

[2] CEI 61025: 1999, Fault tree analysis (FTA). 